\title{
AN IOT BASED FARM MANAGEMENT USING IMAGE PROCESSING
}

\author{
A.Jabeena $^{\# 1}$, G.Suresh ${ }^{* 2}$, S.Swathi ${ }^{\# 3}$, K.Abinaya $^{\# 4}$ \\ \# School of Electronics Engineering, VIT University Vellore, India \\ 12ajabeena@vit.ac.in \\ *Department of Electronics and Communication Engineering, \\ C.Abdul Hakeem College of Engineering \& Technology, Melvisharam, Vellore, India
}

\begin{abstract}
The recent technologies have paved a way to develop and offer advanced services to the stake holders in the agriculture sector.A shift is underway from the monolithic tools to internet based system that can enable more collaboration amongst the stake holders. This paradigm requires technical support from application developers in making specialized services that will interoperate, creating a customisable and sophisticated work environment to the end user. Here in this project, the system is like smart agriculture where it detects insect damge on cultivated crops through monitoring and updates the information about the status of the cultivated crops on the webpage through Ethernet. This system makes automatic irrigation possible. So, in the end it reduces the manpower involved.
\end{abstract}

Keyword- Smart agriculture , insect damage , water irrigation

\section{INTRODUCTION}

Agriculture has been the primary occupation in India. By empowering the agriculture sector,we are actually helping our nation to develope. It is not long since our farmers have been using information systems and technology to keep track of crops. Its common for the farmers to use sophisticated techniques now a days to monitor their crops. A large number of systems are being used to assist and manage farms in an efficient manner. In existing system, the farm house is controlled with only sensors and microcontroller. The following are the sensors that are being used : Temperature sensor and Humidity sensor. The humidity sensor helps in detecting the moisture content of the soil (i.e.) water contents of the soil and drive the water motor. The temperature sensor will detect the farm house temperature and drive the water motor.The disadvantage in the existing system are , it cannot update the status of the cultivated vegetable online and it cannot not find the insect damage on the leaf. In this project, we automatize the farm house maintenance with the help of microcontroller[1]-[4].The microcontroller helps in water irrigation for the plant as well as chemical motor (pesticide). The sensors used in this project are Temperature sensor, Humidity sensor, Soil moisture sensor. The soil moisture sensor which helps to find out soil moisture content (i.e.) if water content of the soil gets reduced it drives the water motor automatically[5]-[8]. The temperature and humidity sensor detects temperature of farm house and also drive the water motor automatically. Matlab is used to find out the insect damage in the leaf using image processing. It will detect automatically insect damage on the leaf and turn on the chemical motor for pesticide of particular plant. The microcontroller will update through Ethernet about cultivation of vegetables[9]-[12].

\section{SYSTEM ARCHITECTURE}

The proposed system makes use of components like the Arduino UNO R3, sensors like humidity sensor, temperature sensor, soil moisturiser sensor, light dependent resistor, relay, Ethernet shield, water motors and power supply unit. The system is of great use and can be afforded by anybody. The system is made in such a way that any one can make use of it efficiently. They themselves can check the soil moisture, temperature and humidity of the soil. Since the amount of light required by crops vary from one crop to another, in order to monitor that, a LDR is used. The relay here is used to control the circuit. There are switches that can operate both mechanically and electrically. By using a software called MATLAB, a predefined set of images are given through a monitoring camera with the help of which the status of the crops are updated onto the webpage. An Ethernet shield has also been used in order to connect the Arduino board to internet. The proposed system is affordable and will be of great help to the farmers.

\section{ARDUINO UNO}

An arduino uno is basically a microcontroller board and its based on ATMEGA328. It basically has 14 output pins which are called as the digital pins and 6 analog pins. It has a 16 mega hertz crystal oscillator, a power jack , USB connection and a reset button. We simply need to connect to a computer using a USB to get started. An Arduino is basically based on Harvard architecture. The program instructions and data have a separate memory. The Atmega328 has 1 KB of EEPROM , 2 KB of SRAM , 32 KB of EEPROM and operates with a clock speed of $16 \mathrm{MHz}$. It has a flash memory for storing code, $0.5 \mathrm{~KB}$ of which is used for bootloader. The important advantage with the Arduino is that the programs can be loaded into the device without the requirement of any programmer to burn program. 


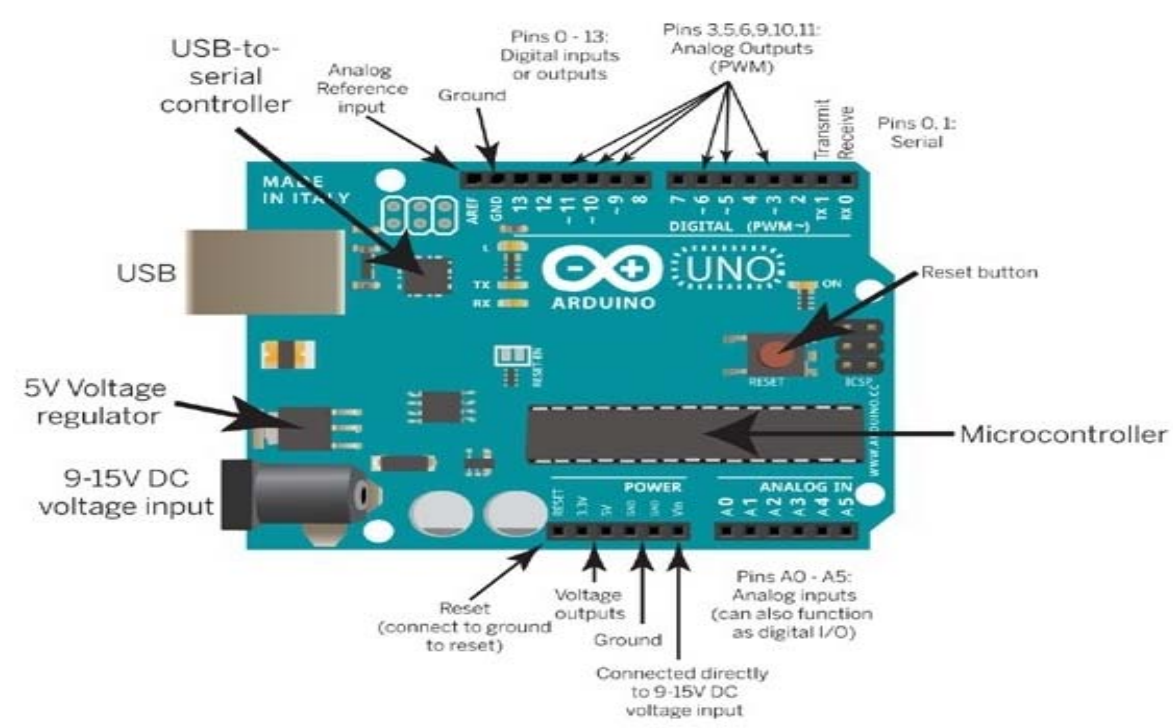

Fig.1. System Architecture of Arduino Uno

\section{TEMPERATURE SENSOR}

Temperature is the most-measured variable in industrial application. A temperature sensor is generally used to convert temperature to a electrical value. Temperature Sensors are used to read and control the temperatures in industries.LM35 IC is a temperaure sensor, where the output voltage is linearly relative to the Celsius. LM35 doesn't need any kind of outside calibration or decoration in order to provide accuracies of $\pm 1 / 4^{\circ} \mathrm{C}$ at room temperature and $\pm 34^{\circ} \mathrm{C}$ over -55 to $+150^{\circ} \mathrm{C}$ temperature range. LM35's, low output impedance, linear output and inherent calibration create an interface to control the circuitry. A humidity sensor is basically a device which can measure the relative humidity in an area. A humidity sensor can be utilized indoors and outdoors. Humidity sensors are mostly available in analog as well as digital forms. This module can convert the relative humidity to voltage. This can be used in weather monitoring applications.

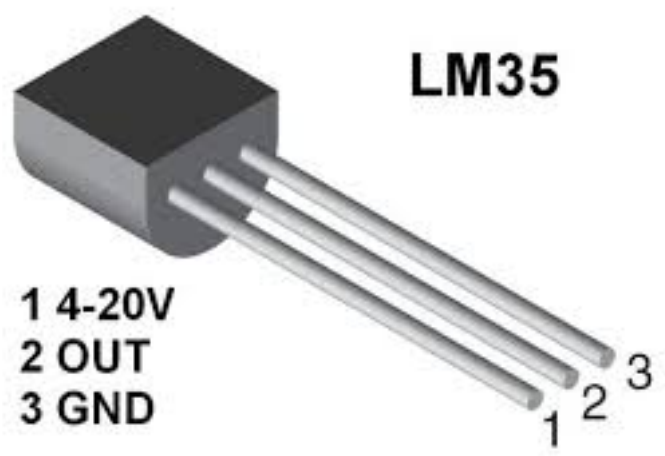

Fig.2 Temperature Sensor

\section{HUMIDITY SENSOR}

A humidity sensor is basically a device which can measure the relative humidity in an area. A humidity sensor can be utilized indoors and outdoors. Humidity sensors are mostly available in analog as well as digital forms. This module can convert the relative humidity to voltage . This can be used in weather monitoring applications.

A. Specifications

- Power Supply: 5V DC

- Power Consumption: <-3.0mA.

- Operating Humidity Range: 30-90\%RH

- Storable Temperature Range: $-30^{\circ} \mathrm{C} \sim 85^{\circ} \mathrm{C}$

- Storable humidity range: within $95 \% \mathrm{RH}$

- Standard output voltage: DC $1.980 \mathrm{mV}$ (at $25^{\circ} \mathrm{C}, 60 \% \mathrm{RH}$ ) 
TABLE I Specification of humidity sensor

\begin{tabular}{|c|c|c|}
\hline Name & Pin & Details \\
\hline GND & 1 & Power Supply Ground \\
\hline$+5 \mathrm{~V}$ & 2 & Power supply Positive input \\
\hline OUT & 3 & Active Low to high analog Output \\
\hline
\end{tabular}

The DHT 11 sensor is basically a low cost sensor. It uses capacitive humidity sensor as well as a thermostat in order to measure the surrounding air and also outputs the digital signal onto the data pin .It is simple in usage, but requires careful timing in order to grab data.

\section{SOIL MOISTURE TEMPERATURE}

A soil moisture sensor is used generally to measure the water content in the soil. The Soil Moisture Sensor is used to detect the moisture level of the soil and to judge if sufficient water is available for the crop.In order to assess the condition of the soil , we need to insert the module into the soil and also adjust the potentiometer on the board so as to adjust sensitivity. The sensor outputs logic HIGH/LOW whenever the moisture content is higher/lower than threshold that has been set by potentiometer.

\section{A. Features}

- $\quad$ Digital output, easy to adjust

- Nickel plating to avoid corrosion

- Working voltage: 3.3V-5V

- $\quad$ On-board LM393 chip

- $\quad$ Dimension of the board: $3.2 \mathrm{~cm} * 1.4 \mathrm{~cm}$

\section{VII.LIGHT DEPENDENT RESISTOR}

Light Dependent Resistors are generally termed as photo resistors. They are dependent on light. Their resistance genereally decreases when light falls on them and increases when they are kept in the dark. This is termed as dark resistance. The dark resistance can be as high as $10^{\wedge} 12$ ohms. Whenever a constant voltage is applied to it, the light intensity increases and the current increases proportionally.

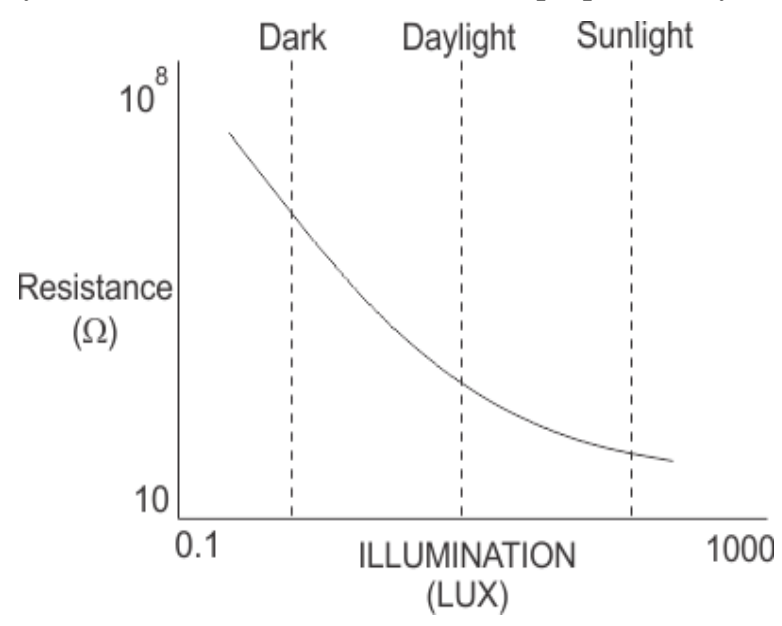

Fig.3 Resistance (vs) Illumination Curve for LDR

\section{DC MOTORS}

A DC motor is used to convert the direct electrical power into mechanical power. It is based on the fact that whenever a current carrying conductor is placed in a magnetic field, then it experiences a mechanical power. Here, in this project,we use 4 motors. Whenever there is an insect damage, a fertilizer motor gets switched on. When the temperature rises, the water motor comes into work. When the LDR rises beyond cutoff then another motor works by spraying water to protect the plants from heat. When the soil moisture sensor detects that the water content of soil is low, then another motor starts working by pouring water to the plants from a tube connected to the motor and it stops after the moisture content of the soil comes back to normal. 


\section{ETHERNET SHIELD}

This is the medium through which the arduino gets the capability to connect to the internet. It is actually based on the wiz net Ethernet chip. It supports up to four simultaneous socket connections. Use the Ethernet library to write sketches which connect to the internet using the shield. The Ethernet shield connects to an Arduino board using long wire-wrap headers which extend through the shield. This keeps the pin layout intact and allows another shield to be stacked on top.We can get connected to the internet using the RJ45 cable:

$>$ It requires an Arduino board (not included).

$>$ It's Operating voltage is $5 \mathrm{~V}$ (which is supplied from Arduino Board).

$>$ Ethernet Controller used here: W5100 with internal 16K buffer.

$>$ The Connection speed is : $10 / 100 \mathrm{Mb}$

$>$ Connection with Arduino is via SPI port.

$>$ The shield contains a considerable number of LEDs.

$>$ PWR: It indicates that the board and shield have been powered.

$>$ LINK: It indicates the presence of athe network link and also flashes whenever the shield transmit or receives data.

$>$ FULLD: It indicates that the network connection is fully duplex.

100M: It indicates the presence of a $100 \mathrm{Mb} / \mathrm{s}$ network connection.

RX: It flashes when the shield receive data.

$>$ TX: It flashes when the shield sends the data.

$>$ COLL: It flashes when network collisions have been detected.

\section{A. MATLAB}

\section{SOFTWARE USED}

Matlab is a fourth genereation programming language. It has been used for a long time in order to make complex mathematical computations easy. They include matrix, interpreting algorithms and many more. They have also been used for interfacing and they are have programs that can be written in C,C++, Java, Fortran, Python.

B. ARDUINO IDE

Arduino IDE is used to interface with arduino through programming which can be uploaded. They generally have files with extension .ino.It has a text editor for writing codes. Programs written here are called as sketches. The programs that are written on the arduino IDE can be uploaded to the arduino board through the arduino USB cable.The message area gives the feedback, displays the output and the errors.

\section{RESULTS}

1. This is the picture (Fig.4.) when crops are monitored for cultivation and once they are ready for cultivation , it displays a message saying "Ready to Cultivate".

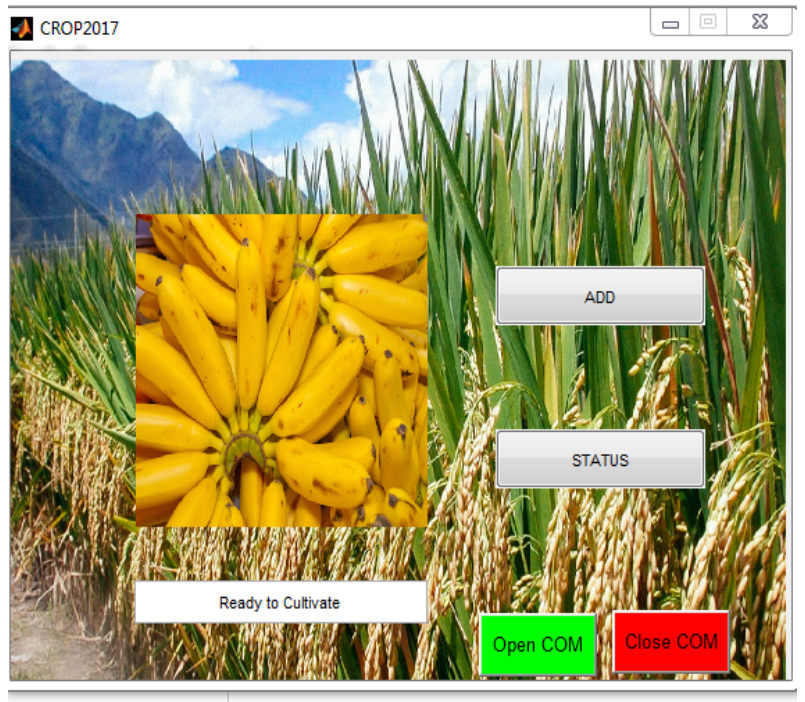

Fig.4 Displays the Status "Ready to Cultivate” 
2. The figure below (Fig.5.) displays the status when the crop is not ready to cultivate.

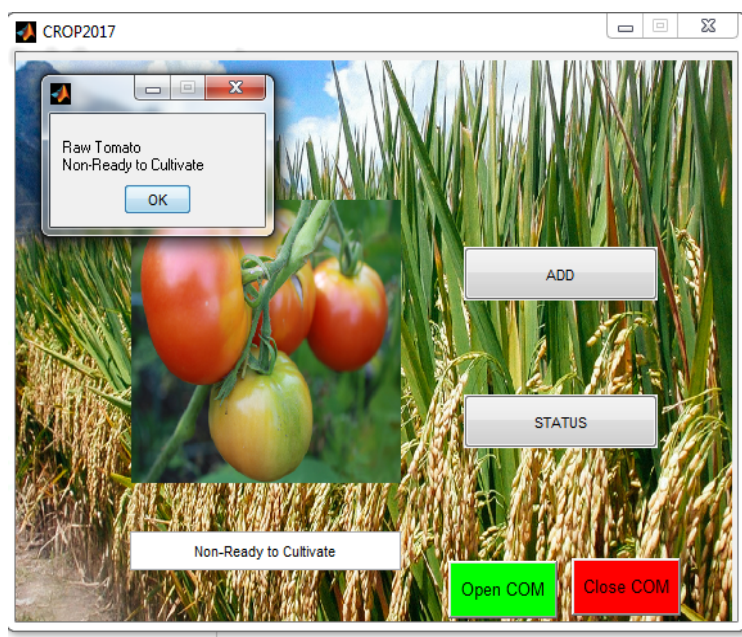

Fig.5. Displays the status not ready to cultivate

3. The figure below( Fig.6.) displays the status of a crop that is affected by the pest and whenever this status is displayed , the motor turns on automatically for fertilizers.

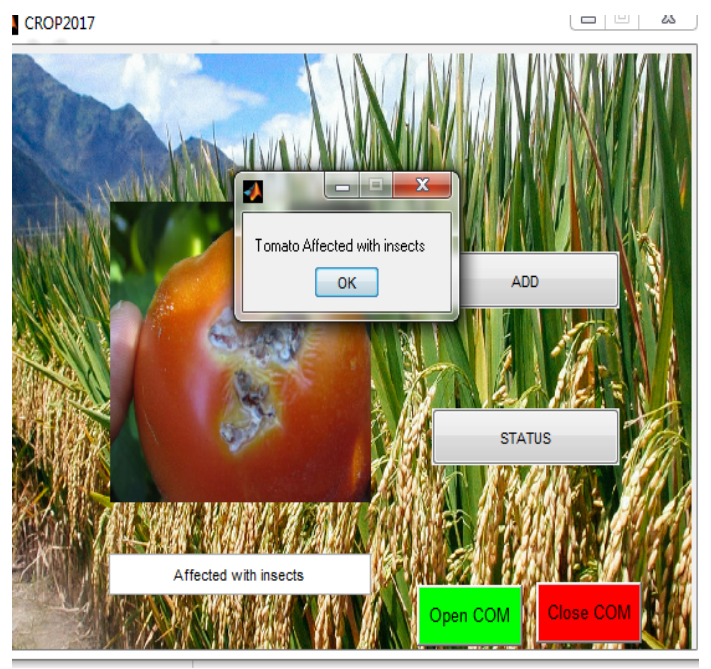

Fig.6. Displays the status of an affected crop

\section{A. Webpage}

The following figure (Fig.7.) displays the upadated status of the crops on webpage.

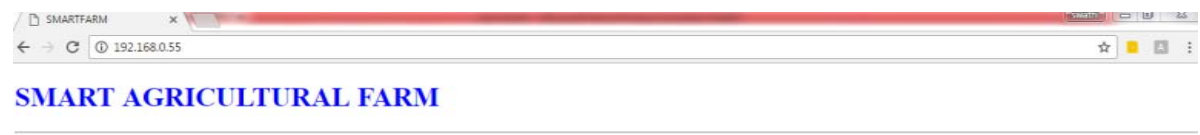

\begin{tabular}{|c|c|c|c|}
\hline 5 sono & Parameter & Value & Status \\
\hline 1 & Tenpersture & 2989 & Normal \\
\hline 2 & Soil Meosture & 344 & Metor ion \\
\hline 3 & LDR senisers & 37 & Sura Lighth is Nonnal \\
\hline 4 & Hamidity Sensor & 96 & Normal \\
\hline 5 & Stanus & 1 & Ready for Ciltrvation \\
\hline
\end{tabular}




\section{XII.CONCLUSION}

This IOT based farm management system is a very reliable and a very efficient system. It can really make manpower less and can be more helpful for people in modern cities as the status of the crops are updated on the webpage that they can access from anywhere and at anytime.There is no necessity for them to continuously keep monitoring the crops.With the proposed system, using the sensors, the temperature, light intensity, soil moisture as well as humidity are monitored and whenever the value seems abnormal the respective motors gets switched on. More over this system can detect the damage caused by the insects and automatically turns on the fertilizer motor that makes the system even more efficient and reliable.

\section{REFERENCES}

[1] Junyan Ma, Xingshe Zhou, Zhigang Li, "Connecting Agriculture to the Internet of Things through Sensor Networks", Internet of Things (iThings/CPSCom) 2011 International Conference on and 4th International Conference on Cyber Physical and Social Computing, pp. 184-187, Oct 2011.

[2] Duan Yan-e, "Design of Intelligent Agriculture Management Information System Based on IoT", Intelligent Computation Technology and Automation (ICICTA) 2011 International Conference, vol. 1, pp. 1045-1049, March 2011.

[3] Meonghun Lee, Sunchon Nat, Jeonghwan Hwang, "Agricultural Production System Based on IoT", Computational Science and Engineering (CSE) 2013 IEEE 16th International Conference, pp. 833-836, Dec 2013.

[4] M V Latte Sushila Shidnal ,’Multiple Nutrient Deficiency Detection in Paddy Leaf Images using Color and Pattern Analysis”,In: International conference on communication and signal processing, pp. 1007-1010, 2016.

[5] V. Ram, H. Vishal, S. Dhanalakshmi, P. M eenakshiVidya, "Regulation of water in agriculture field using Internet Of Things", Technological Innovation in ICT for Agriculture and rural development (TIAR), IEEE, pp. 112-115, 2015.

[6] Rui Jiang, Sun Danpeng, "Architecture Design of the Internet of Things based on Cloud Computing", In: Measuring Technology and Mechatronics Automation (ICMTMA) 2015 Seventh International Conference on, pp. 206-209, 2015.

[7] Balaji Bhanu, Raghava Rao, J.V.N. Ramesh, Mohammed Ali Hussain, "Agriculture Field Monitoring and Analysis using Wireless Sensor Networks for improving Crop Production", Eleventh International Conference on Wireless and Optical Communications Networks (WOCN), 2014.

[8] LIU Dan, Cao Xin, Huang Chongwei, JI Liang Liang, "Intelligent agent greenhouse environment monitoring system based on IOT technology", In: International Conference on Intelligent Transportation Big Data \& Smart City, 2015.

[9] Joseph Haule, Kisangiri Michael, "Deployment of wireless sensor networks (WSN) in automated irrigation management and scheduling systems: a review", Science Computing and Telecommunications (PACT), 2014.

[10] Rodolfo Bongiovanni, Jess Lowenberg-DeBoer, "Precision agriculture and sustainability", Precision agriculture, vol. 5, no. 4, pp. 359387, 2004.

[11] Neil Gershenfeld, Raffi Krikorian, Danny Cohen, "The Internet of Things", Scientific American, vol. 291, no. 4, pp. 76-81, 2004.

[12] Kerry Taylor et al., "Farming the web of things", Intelligent Systems, vol. 28, no. 6, pp. 12-19, 2013.

\section{AUTHOR PROFILE}

A.Jabeena is an associate professor in School of Electronics Engineering ,VIT University,Vellore,Tamilnadu, India. Her research interest includes Optical communication, Free space and Visible light communication.

G.Suresh is an associate professor in Department of Electronics and Communication Engineering, C.Abdul Hakeem College of Engineering \& Technology, Melvisharam, Vellore, Tamilnadu, India.

S.Swathi is a B.Tech, ECE students from School of Electronics Engineering ,VIT University, Vellore, Tamilnadu, India.

K.Abinaya is a B.Tech , ECE students from School of Electronics Engineering ,VIT University, Vellore, Tamilnadu, India. 